\title{
S-Approximation: A New Approach to Algebraic Approximation
}

\author{
M. R. Hooshmandasl, ${ }^{1,2}$ A. Shakiba, ${ }^{1,2}$ A. K. Goharshady, ${ }^{1,2}$ and A. Karimi ${ }^{3}$ \\ ${ }^{1}$ Department of Computer Science, Yazd University, Yazd, Iran \\ ${ }^{2}$ The Laboratory of Quantum Information Processing, Yazd University, Yazd, Iran \\ ${ }^{3}$ Mathematics Department, Western Illinois University, IL, USA
}

Correspondence should be addressed to M. R. Hooshmandasl; hooshmandasl@yazd.ac.ir

Received 16 October 2013; Accepted 14 April 2014; Published 28 April 2014

Academic Editor: Zhan Zhou

Copyright (C) 2014 M. R. Hooshmandasl et al. This is an open access article distributed under the Creative Commons Attribution License, which permits unrestricted use, distribution, and reproduction in any medium, provided the original work is properly cited.

We intend to study a new class of algebraic approximations, called $S$-approximations, and their properties. We have shown that $S$-approximations can be used for applied problems which cannot be modeled by inclusion based approximations. Also, in this work, we studied a subclass of $S$-approximations, called $S_{\mathscr{M}}$-approximations, and showed that this subclass preserves most of the properties of inclusion based approximations but is not necessarily inclusionbased. The paper concludes by studying some basic operations on $S$-approximations and counting the number of $S$-min functions.

\section{Introduction}

Uncertainty is often present in real-life applications. Uncertainty in noncrisp sets is characterized by nonempty boundary regions, in which nothing can be said about their elements with certainty. In classical set theory, a subset $A$ of a universe $U$ induces a partition $\{A, U-A\}$ over that universe. This partition can be interpreted as a knowledge on elements of $U$; that is, elements in $A$ are indiscernible to each other and also the same thing holds for items in $U-A$. This knowledge may be improved to another partition, for example, $\mathscr{P}$, whose items in each partition are indiscernible to each other. In consequence, for a subset $A$ of $U$, the problem of whether $x$ belongs to $A$ or not, with respect to knowledge $\mathscr{P}$, may become undecidable; that is, some elements indiscernible to $x$ with respect to knowledge $\mathscr{P}$ may be in $A$, whereas some other indiscernible elements to $x$ with knowledge $\mathscr{P}$ may not belong to $A$. To cope with such uncertainty, some tools were invented such as the Dempster-Shafer theory of evidence [1], theory of fuzzy sets [2-5], and theory of rough sets [6-8]. Rough set theory and fuzzy set theory are two independent approaches for uncertainty. There is a connection between rough set theory and Dempster-Shafer theory. Strictly speaking, lower and upper approximations of rough set theory correspond to the inner and outer reductions from Dempster-Shafer theory [9].
Rough set theory and its generalizations are all based on the inclusion relation $[7,8,10-15]$, which is a limitation in approximations. In this work, we introduce a new concept named $S$-approximation set. This concept is independent of inclusion relation and contains rough sets and their generalizations as special cases. We provide some examples of approximations using this new concept, which cannot be obtained by rough set theory.

This paper is organized as follows. The notion of $S$ approximation sets is proposed in Section 2, followed by considering some operations on them. The definition of $S_{\mathscr{M}}$ conditioned rough sets is proposed in Section 3 and the number of such sets is counted. Then we conclude the paper.

\section{2. $\mathcal{S}$-Approximation}

In this section, with regard to Dempster's multivalued mappings [16], we propose a new mathematical approach to study approximation spaces and we will show that this concept can be independent of inclusion relations and the rough set and its generalizations are all special cases of this concept.

Definition 1. An $\mathcal{S}$-approximation is the quadruple $G=$ $(U, W, T, S)$, where $U$ and $W$ are finite nonempty sets, $T$ is a 
mapping of the form $T: U \rightarrow P^{\star}(W)$, and $S$ is a mapping of the form $S: P^{\star}(W) \times P^{\star}(W) \rightarrow\{0,1\}$.

For a nonempty subset $X$ of $W$, the upper and lower approximations of $X$ are defined as follows:

$$
\begin{aligned}
& \overline{\operatorname{apr}}_{G}(X)=\left\{x \in U \mid S\left(T(x), X^{c}\right)=0\right\}, \\
& \underline{\operatorname{apr}}_{G}(X)=\{x \in U \mid S(T(x), X)=1\},
\end{aligned}
$$

where $X^{c}$ is the complement of $X$ with respect to $W$.

Example 2. Let $U$ and $W$ be nonempty finite sets and let $R$ be a relation from $U$ to $W$. We define $G=(U, W, T, S)$, where $T$ and $S$ are defined as

$$
\begin{gathered}
T(x)=[x]_{R}=\{y \in W \mid x R y\}, \\
S(A, B)= \begin{cases}1, & A \subseteq B, \\
0, & \text { otherwise. }\end{cases}
\end{gathered}
$$

The upper approximation of $X$ with respect to $G, \overline{\operatorname{apr}}_{G}(X)$, is equal to $\bar{R}(X)$, the upper approximation of set $X$ with respect to $R$ in rough set, since

$$
\begin{aligned}
\overline{\operatorname{apr}}_{G}(X) & =\left\{x \in U \mid S\left(T(x), X^{c}\right)=0\right\} \\
& =\left\{x \in U \mid T(x) \nsubseteq X^{c}\right\} \\
& =\{x \in U \mid T(x) \cap X \neq \emptyset\} \\
& =\left\{x \in U \mid[x]_{R} \cap X \neq \emptyset\right\}=\bar{R}(X) .
\end{aligned}
$$

Similarly, we can show that $\operatorname{apr}_{G}(X)$ is equal to $\underline{R}(X)$, which is the lower approximation of set $X$ with respect to $R$ in rough set.

Example 3. The pair $(V, H)$, where $V$ is a finite nonempty set of vertices and $H$ is a collection $\left\{E_{1}, \ldots, E_{m}\right\}$ of subsets of $V$, is called a simple hypergraph if these two conditions hold:

(i) $\cup_{i=1}^{m} E_{i}=V$,

(ii) $E_{i} \subseteq E_{j}$ implies $i=j$.

Let us define the $S$-approximation $G=\left(\mathscr{I}_{m}, V, T, S\right)$ such that $\mathscr{I}_{m}=\{1, \ldots, m\}, T(i)=E_{i}$, and $S$ is the inclusion function.

A subset $K$ of $V$ is called a transversal of $(V, H)$ if and only if $K \cap E_{i} \neq \emptyset$ for each $1 \leq i \leq m$. It is easy to observe that $K$ is a transversal of $(V, H)$ if and only if $\overline{\operatorname{apr}}_{G}(K)=\mathscr{I}_{m}$.

Remark. For the sake of simplicity, we will use $\underline{G}(X)$ and $\bar{G}(X)$ instead of $\underline{\operatorname{apr}}_{G}(X)$ and $\overline{\operatorname{apr}}_{G}(X)$, respectively.

\subsection{Operations on S-Approximation}

Definition 4. Suppose $G=(U, W, T, S)$ is an $S$-approximation. One defines $H=(U, W, T, 1-S)$ as the complement of $G$.
Proposition 5. Let $G=(U, W, T, S)$ be an $S$-approximation and $H=\left(U, W, T, S^{\prime}=1-S\right)$ its complement. Also suppose that $X \subseteq W$. Then one has

$$
\begin{aligned}
\bar{G}(X) & =\left\{x \in U \mid S\left(T(x), X^{c}\right)=0\right\} \\
& =\left\{x \in U \mid S^{\prime}\left(T(x), X^{c}\right)=1\right\} \\
& =\underline{H}\left(X^{c}\right), \\
\underline{G}(X) & =\{x \in U \mid S(T(x), X)=1\} \\
& =\left\{x \in U \mid S^{\prime}(T(x), X)=0\right\} \\
& =\bar{H}\left(X^{c}\right) .
\end{aligned}
$$

Definition 6. Let $W$ be a finite nonempty set. Consider the following:

$$
\Omega_{T}(W)=\left\{S \mid S: P^{\star}(W) \times P^{\star}(W) \longrightarrow\{0,1\}\right\},
$$

and $S_{1}, S_{2} \in \Omega_{T}(W)$. Then one defines the following:

$$
\begin{aligned}
& \left(S_{1} \wedge S_{2}\right)(A, B)=S_{1}(A, B) \wedge S_{2}(A, B), \\
& \left(S_{1} \vee S_{2}\right)(A, B)=S_{1}(A, B) \vee S_{2}(A, B),
\end{aligned}
$$

where $A$ and $B$ are arbitrary subsets of $W$.

Definition 7. Let $G_{1}=\left(U, W, T, S_{1}\right)$ and $G_{2}=\left(U, W, T, S_{2}\right)$ be two $S$-approximations. One defines $G_{1} \wedge G_{2}$ and $G_{1} \vee G_{2}$ as

$$
\begin{aligned}
& G_{1} \wedge G_{2}=\left(U, W, T, S_{1} \wedge S_{2}\right), \\
& G_{1} \vee G_{2}=\left(U, W, T, S_{1} \vee S_{2}\right),
\end{aligned}
$$

respectively.

Proposition 8. Let $G_{1}=\left(U, W, T, S_{1}\right), G_{2}=\left(U, W, T, S_{2}\right)$, and $G=\left(U, W, T, S=S_{1} \vee S_{2}\right)$ be $S$-approximations. Then

$$
\begin{gathered}
\bar{G}(X) \quad \\
=\left\{x \in U \mid S\left(T(x), X^{c}\right)=0\right\} \\
=\left\{x \in U \mid S_{1}\left(T(x), X^{c}\right)\right. \\
\left.=0 \wedge S_{2}\left(T(x), X^{c}\right)=0\right\} \\
=\left\{x \in U \mid S_{1}\left(T(x), X^{c}\right)=0\right\} \\
\quad \cap\left\{x \in U \mid S_{2}\left(T(x), X^{c}\right)=0\right\} \\
=\overline{G_{1}}(X) \cap \overline{G_{2}}(X), \\
=\{x \in U \mid S(T(x), X)=1\} \\
=\left\{x \in U \mid S_{1}(T(x), X)=1 \vee S_{2}(T(x), X)=1\right\} \\
=\left\{x \in U \mid S_{1}(T(x), X)=1\right\} \\
\cup\left\{x \in U \mid S_{2}(T(x), X)=1\right\} \\
=\underline{G_{1}}(X) \cup \underline{G_{2}}(X) .
\end{gathered}
$$


Similarly, for $G^{\prime}=\left(U, W, T, S_{1} \wedge S_{2}\right)$, it can be shown that the following relations hold:

$$
\begin{aligned}
& \overline{G^{\prime}}(X)=\overline{G_{1}}(X) \cup \overline{G_{2}}(X), \\
& \underline{G^{\prime}}(X)=\underline{G_{1}}(X) \cap \underline{G_{2}}(X) .
\end{aligned}
$$

\section{3. $S_{\mathscr{M}}$-Approximations}

In this section, we introduce and discuss a condition on $S$ which is sufficient for the properties in Proposition 13 to hold. These properties are sometimes vital for many applications.

In the next example we illustrate the fact that it is not necessary for $S$ to be the inclusion function in order to satisfy the properties stated in Proposition 13.

Example 9. For arbitrary subsets $A, B \subseteq W$, define the following:

$$
S(A, B)= \begin{cases}1, & \text { if } A \cup B=W, \\ 0, & \text { otherwise. }\end{cases}
$$

It can be verified that this function is not the same as the inclusion function, but the properties of Proposition 13 hold for the $S$-approximation $G=(U, W, T, S)$ with arbitrary chosen $U, W$, and $T$.

The reason why $G$ in the above example satisfies the properties of Proposition 13 is that its $S$ function meets the $S$-min condition introduced below.

Definition 10 ( $S$-min condition). Let $G=(U, W, T, S)$ be an $S$-approximation. One says $S: P^{\star}(W) \times P^{\star}(W) \rightarrow\{0,1\}$ is a function in $S_{\mathscr{M}}$ class if it satisfies

$$
S(A, B \cap C)=\min \{S(A, B), S(A, C)\},
$$

for arbitrary nonempty subsets $A, B$, and $C$ of $W$. One says an $S$-approximation $G=\left(U, W, T, S^{\prime}\right)$ is an $S_{\mathscr{M}}$-approximation if $S^{\prime}$ belongs to the $S_{\mathscr{M}}$ class.

Remark 11. The inclusion function does belong to the $S_{\mathscr{M}}$ class but there are other noninclusion functions in this class as well.

Example 12. For arbitrary subsets $A, B$, and $X$ of $W$, define the following:

$$
S_{X}(A, B)= \begin{cases}1, & \text { if } X \subseteq A \cap B, \\ 0, & \text { otherwise }\end{cases}
$$

It is easy to check that $S_{X}$ is a noninclusion member of the $S_{\mathscr{M}}$ class.

Proposition 13. Let $G=(U, W, T, S)$ be an $S_{\mathscr{M}^{-}}$ approximation. For all $A, B \subseteq W$ and $x \in U$, the following hold:

(1) $A \subseteq B$ implies that, for all $X \subseteq W, S\left(X, B^{c}\right) \leq S\left(X, A^{c}\right)$,

(2) $\max \{\mathrm{S}(\mathrm{T}(\mathrm{x}), \mathrm{A}), \mathrm{S}(\mathrm{T}(\mathrm{x}), \mathrm{B})\} \leq \mathrm{S}(\mathrm{T}(\mathrm{x}), \mathrm{A} \cup \mathrm{B})$,
(3) $\bar{G}(A \cup B)=\bar{G}(A) \cup \bar{G}(B)$,

(4) $\underline{G}(A \cap B)=\underline{G}(A) \cap \underline{G}(B)$,

(5) $A \subseteq B$ implies that $\underline{G}(A) \subseteq \underline{G}(B)$,

(6) $A \subseteq B$ implies that $\bar{G}(A) \subseteq \bar{G}(B)$,

(7) $\underline{G}(A) \cup \underline{G}(B) \subseteq \underline{G}(A \cup B)$,

(8) $\bar{G}(A \cap B) \subseteq \bar{G}(A) \cap \bar{G}(B)$,

(9) $\underline{G}(A)=\left(\bar{G}\left(A^{c}\right)\right)^{c}$,

(10) $\bar{G}(A)=\left(\underline{G}\left(A^{c}\right)\right)^{c}$.

Proof. (1) For the first property, note that $A \subseteq B$ so $B^{c}=$ $B^{c} \cap A^{c}$. Hence, for all $X \subseteq W$, we have $S\left(X, B^{c}\right)=S\left(X, B^{c} \cap\right.$ $\left.A^{c}\right)=\min \left\{S\left(X, B^{c}\right), S\left(X, A^{c}\right)\right\}$, which implies that $S\left(X, B^{c}\right) \leq$ $S\left(X, A^{c}\right)$.

(2) For this property, we have

$$
\begin{aligned}
S(T(x), A) & =S(T(x), A \cap(A \cup B)) \\
& =\min \{S(T(x), A), S(T(x), A \cup B)\},
\end{aligned}
$$

which implies that

$$
S(T(x), A) \leq S(T(x), A \cup B) .
$$

By a similar argument for $B$, it can be shown that

$$
S(T(x), B) \leq S(T(x), A \cup B) .
$$

By combining these inequalities,

$\max \{S(T(x), A), S(T(x), B)\} \leq S(T(x), A \cup B)$.

(3) Consider the following:

$$
\begin{aligned}
\bar{G}(A \cup B) & =\left\{x \in U \mid S\left(T(x), A^{c} \cap B^{c}\right)=0\right\} \\
& =\left\{x \in U \mid \min \left\{S\left(T(x), A^{c}\right), S\left(T(x), B^{c}\right)\right\}=0\right\} \\
& =\bar{G}(A) \cup \bar{G}(B) .
\end{aligned}
$$

(4) Consider the following:

$$
\begin{aligned}
\underline{G}(A \cap B) & =\{x \in U \mid S(T(x), A \cap B)=1\} \\
& =\{x \in U \mid S(T(x), A)=1, S(T(x), B)=1\} \\
& =\underline{G}(A) \cap \underline{G}(B) .
\end{aligned}
$$

(5) Consider the following:

$$
\begin{aligned}
\underline{G}(A) & =\underline{G}(A \cap B) \quad(\text { since } A \subseteq B) \\
& =\underline{G}(A) \cap \underline{G}(B) \quad(\text { by property }(1)) .
\end{aligned}
$$

which implies that $\underline{G}(A) \subseteq \underline{G}(B)$.

(6) Consider the following:

$$
\begin{aligned}
\bar{G}(A) & =\left\{x \in U \mid S\left(T(x), A^{c}\right)=0\right\} \\
& \subseteq\left\{x \in U \mid S\left(T(x), B^{c}\right)=0\right\} \quad \text { (by property (1)) } \\
& =\bar{G}(B) .
\end{aligned}
$$


(7) Consider the following:

$$
\begin{aligned}
& \underline{G}(A) \cup \underline{G}(B) \\
& =\{x \in U \mid S(T(x), A)=1 \text { or } S(T(x), B)=1\} \\
& =\{x \in U \mid \max \{S(T(x), A), S(T(x), B)\}=1\} \\
& \subseteq\{x \in U \mid S(T(x), A \cup B)=1\} \quad \text { (by property (1)) } \\
& \subseteq \underline{G}(A \cup B) .
\end{aligned}
$$

(8) Consider the following:

$$
\begin{aligned}
& \bar{G}(A \cap B) \\
& =\left\{x \in U \mid S\left(T(x), A^{c} \cup B^{c}\right)=0\right\} \\
& \subseteq\{x \in U \mid \max \{S(T(x), A), S(T(x), B)\}=0\}
\end{aligned}
$$$$
\text { (by property (2)) }
$$

$$
\begin{aligned}
& =\{x \in U \mid S(T(x), A)=0, S(T(x), B)=0\} \\
& =\bar{G}(A) \cap \bar{G}(B)
\end{aligned}
$$

The proof of properties (9) and (10) is entirely straightforward.

In the next example, we show that, in $S$-rough sets, it is not always the case that $\underline{G}(A) \subseteq \bar{G}(A)$, although this property always holds in Pawlak's rough sets.

Example 14. Suppose $G=(U, W, T, S)$ is an $S_{\mathscr{M}^{-}}$ approximation, where

$$
S(A, B)= \begin{cases}1, & A \cup B=W, \\ 0, & \text { otherwise }\end{cases}
$$

$U=\{a\}$, and $T(a)=W=\{1,2\}$.

In this case $\bar{G}(\{1\})=\{x \in U \mid T(x) \cup\{2\} \neq W\}=\emptyset$, while

$$
\underline{G}(\{1\})=\{x \in U \mid T(x) \cup\{1\}=W\}=\{a\},
$$

so $\underline{G}(\{1\}) \nsubseteq \bar{G}(\{1\})$.

\subsection{Cardinality of the $S_{\mathscr{M}}$ Class}

Definition 15. Let $W$ be a nonempty finite set. A function $f$ : $P^{\star}(W) \rightarrow\{0,1\}$ is said to be minimizing if, for each $A, B \subseteq$ $W$,

$$
f(A \cap B)=\min \{f(A), f(B)\} .
$$

Lemma 16. Let $f: P^{\star}(W) \rightarrow\{0,1\}$ be a minimizing function. For each $A, B \subseteq W$, if $A \subseteq B$, then $f(A) \leq f(B)$.

Proof. Since $A \subseteq B, A=A \cap B$, so by definition $f(A)=$ $\min \{f(A), f(B)\}$ which implies that $f(A) \leq f(B)$.

Lemma 17. Let $G=(U, W, T, S)$ be an $S_{M}$-approximation and let $|W|=n$. One labels the nonempty subsets of $W$ as $\left\{A_{1}, \ldots, A_{2^{n}-1}\right\}$. Then there exist minimizing functions $\left\{f_{1}, \ldots, f_{2^{n}-1}\right\}$ of the form $f_{i}: P^{\star}(W) \rightarrow\{0,1\}$ such that, for every $B \subseteq W$ and $1 \leq i \leq n, S\left(A_{i}, B\right)=$ $f_{i}(B)$.

Proof. It is straightforward.

Lemma 17 leads us towards counting the number and finding the structure of minimizing $f$ s.

Definition 18. Let $f: P^{\star}(W) \rightarrow\{0,1\}$ be a minimizing function. A nonempty subset $\omega$ of the set $W$ is called an atom of $f$ if and only if $f(\omega)=1$ and, for each proper nonempty subset of $\omega$ such as $\eta, f(\eta)=0$.

Proposition 19. Let $f: P^{\star}(W) \rightarrow\{0,1\}$ be a minimizing function and $\omega_{1}$ and $\omega_{2}$ two nonidentical atoms of $f$. Then $\omega_{1} \cap$ $\omega_{2}=\emptyset$.

Proof. Let $\omega=\omega_{1} \cap \omega_{2}$; then, since $\omega_{1}$ and $\omega_{2}$ are nonidentical atoms, $\omega \neq \omega_{1}$ and $\omega \neq \omega_{2}$. Suppose that $\omega \neq \emptyset$. By Definition $15, f(\omega)=\min \left\{f\left(\omega_{1}\right), f\left(\omega_{2}\right)\right\}=1$, so $\omega$ is a proper subset of $\omega_{1}$ and $\omega_{2}$ and $f(\omega)=1$ which is a contradiction with Definition 18.

Proposition 20. Let $f: P^{\star}(W) \rightarrow\{0,1\}$ be a minimizing function and $Y$ the set of all atoms of $f$. Then, for a subset $X$ of $W, f(X)=1$ if and only if there exists $\omega \in \Upsilon$ such that $\omega \subseteq X$.

Proof. It is obvious that if $f(X)=1$, then at least one of the subsets of $X$ is an atom. On the other hand, let $\omega \subseteq X$ be an atom of $f$; then, by Lemma $16, f(X)=1$.

Proposition 21. Let $f: P^{\star}(W) \rightarrow\{0,1\}$ be a minimizing function, $\Upsilon$ the set of all atoms of $f$, and $|\Upsilon| \geq 2$. Then, for each $x \in W,\{x\}$ is an atom of $f$.

Proof. Let $\omega_{1}$ and $\omega_{2}$ be two different atoms of $f$. Define $A=$ $\omega_{1} \cup\{x\}$ and $B=\omega_{2} \cup\{x\}$; then, by Proposition 20, $f(A)=$ $f(B)=1$ and, by Proposition $19, A \cap B=\{x\} . f$ is minimizing so $f(\{x\})=f(A \cap B)=\min \{f(A), f(B)\}=1$; hence $\{x\}$ is an atom of $f$.

By previous propositions, it is clear that we have either no atoms, exactly one atom, or an atom per element.

Proposition 22. Define $F$ as the set of all minimizing functions $f$ of the form $f: P^{\star}(W) \rightarrow\{0,1\}$, where $|W|=n \geq 2$. Then the total number of elements in $F$ is equal to $2^{n}+1$.

Proof. To count the number of elements in $F$, we consider these three cases for each $f \in F$.

(i) $f$ does not have any atoms: in this case, $f$ is determined uniquely. ( $f(X)=0$ for each nonempty $X \subseteq$ W.)

(ii) Each unary subset of $f$ forms an atom: in this case, $f$ is determined uniquely too. $(f(X)=1$ for each nonempty $X \subseteq W$.)

(iii) There is exactly one atom: in this case, we can choose $2^{n}-1$ different atoms (excluding $\emptyset$ ) giving us $2^{n}-1$ 
different $f$ s. In case $n=1$, this case is a repetition of the previous case.

Proposition 23. Let $W$ be a nonempty finite set of size $n \geq 2$. The total number of different functions $S: P^{\star}(W) \times P^{\star}(W) \rightarrow$ $\{0,1\}$ which belong to the $S$-min class is equal to $\left(2^{n}+1\right)^{2^{n}-1}$.

Proof. This number can be obtained using the multiplication principle, Lemma 17, and Proposition 22.

\section{Conclusion}

In this paper, we proposed a new class of algebraic approximation, called $S$-approximation sets. Corresponding to the problem under consideration, we can define the elements of $S$-approximation set for obtaining the approximations. Moreover, we investigated the properties of a subclass of $S$ approximation sets, $S_{\mathscr{M}}$-approximation sets. We have shown that this subclass preserves most of the properties of inclusion based approximations but is not necessarily inclusion based. Finally, we have considered some basic operations on $S$ approximation sets and counted the number of functions which have the $S_{\mathscr{M}}$ property.

\section{Conflict of Interests}

The authors declare that there is no conflict of interests regarding the publication of this paper.

\section{References}

[1] G. Shafer, A Mathematical Theory of Evidence, vol. 1, Princeton University Press, Princeton, NJ, USA, 1976.

[2] L. A. Zadeh, "simple view of the dempster-shafer theory of evidence and its implication for the rule of combination," $A I$ Magazine, vol. 7, no. 2, pp. 85-90, 1986.

[3] L. A. Zadeh, "A computational theory of dispositions," in Proceedings of the 10th International Conference on Computational Linguistics, pp. 312-318, Association for Computational Linguistics, 1984.

[4] L. A. Zadeh, "The role of fuzzy logic in the management of uncertainty in expert systems," Fuzzy Sets and Systems, vol. 11, no. 1-3, pp. 197-198, 1983.

[5] L. A. Zadeh, "Fuzzy sets," Information and Computation, vol. 8, pp. 338-353, 1965.

[6] Z. Pawlak, Rough Sets: Theoretical Aspects of Reasoning About Data, Theory and decision library: System theory, knowledge engineering, and problem solving, Kluwer Academic Publishers, 1991.

[7] Z. Pawlak, "Rough classification," International Journal of ManMachine Studies, vol. 20, no. 5, pp. 469-483, 1984.

[8] Z. Pawlak, "Rough sets," International Journal of Computer and Information Sciences, vol. 11, no. 5, pp. 341-356, 1982.

[9] J. W. Grzymala-Busse, "Knowledge acquisition under uncertainty-a rough set approach," Journal of Intelligent and Robotic Systems, vol. 1, no. 1, pp. 3-16, 1988.

[10] Z. Pawlak, S. K. M. Wong, and W. Ziarko, "Rough sets: probabilistic versus deterministic approach," International Journal of Man-Machine Studies, vol. 29, no. 1, pp. 81-95, 1988.
[11] Z. Pawlak and A. Skowron, "Rough membership functions," in Advances in the Dempster-Shafer Theory of Evidence, R. Yager, M. Fedrizzi, and J. Kacprzyk, Eds., pp. 251-271, John Wiley \& Sons, 1994.

[12] Y. Y. Yao and T. Y. Lin, "Generalization of rough sets using modal logic," Intelligent Automation and Soft Computing, vol. 2, no. 2, pp. 103-120, 1996.

[13] Y. Y. Yao, "On generalizing rough set theory," in Rough Sets, Fuzzy Sets, Data Mining, and Granular Computing, G. Wang, Q. Liu, Y. Yao, and A. Skowron, Eds., vol. 2639 of Lecture Notes in Computer Science, pp. 44-51, Springer, Berlin, Germany, 2003.

[14] Y. Y. Yao, "Two views of the theory of rough sets in finite universes," International Journal of Approximate Reasoning, vol. 15, no. 4, pp. 291-317, 1996.

[15] Y. Y. Yao, S. K. M. Wong, and T. Y. Lin, "A review of rough set models," in Rough Sets and Data Mining, pp. 47-75, Springer, 1996.

[16] A. P. Dempster, "Upper and lower probabilities induced by a multivalued mapping," The Annals of Mathematical Statistics, vol. 38, no. 2, pp. 325-339, 1967. 


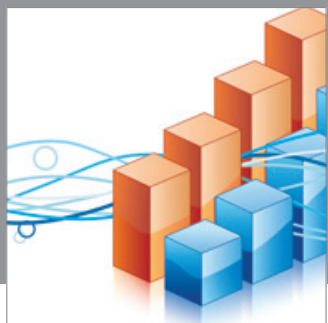

Advances in

Operations Research

mansans

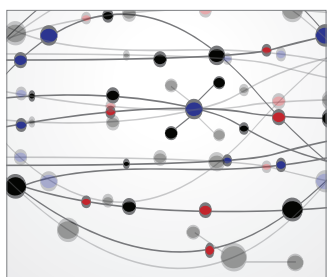

The Scientific World Journal
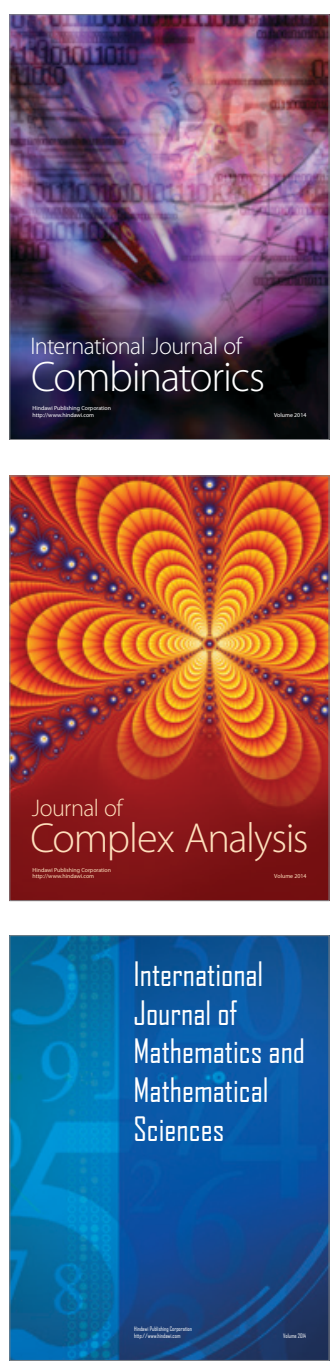
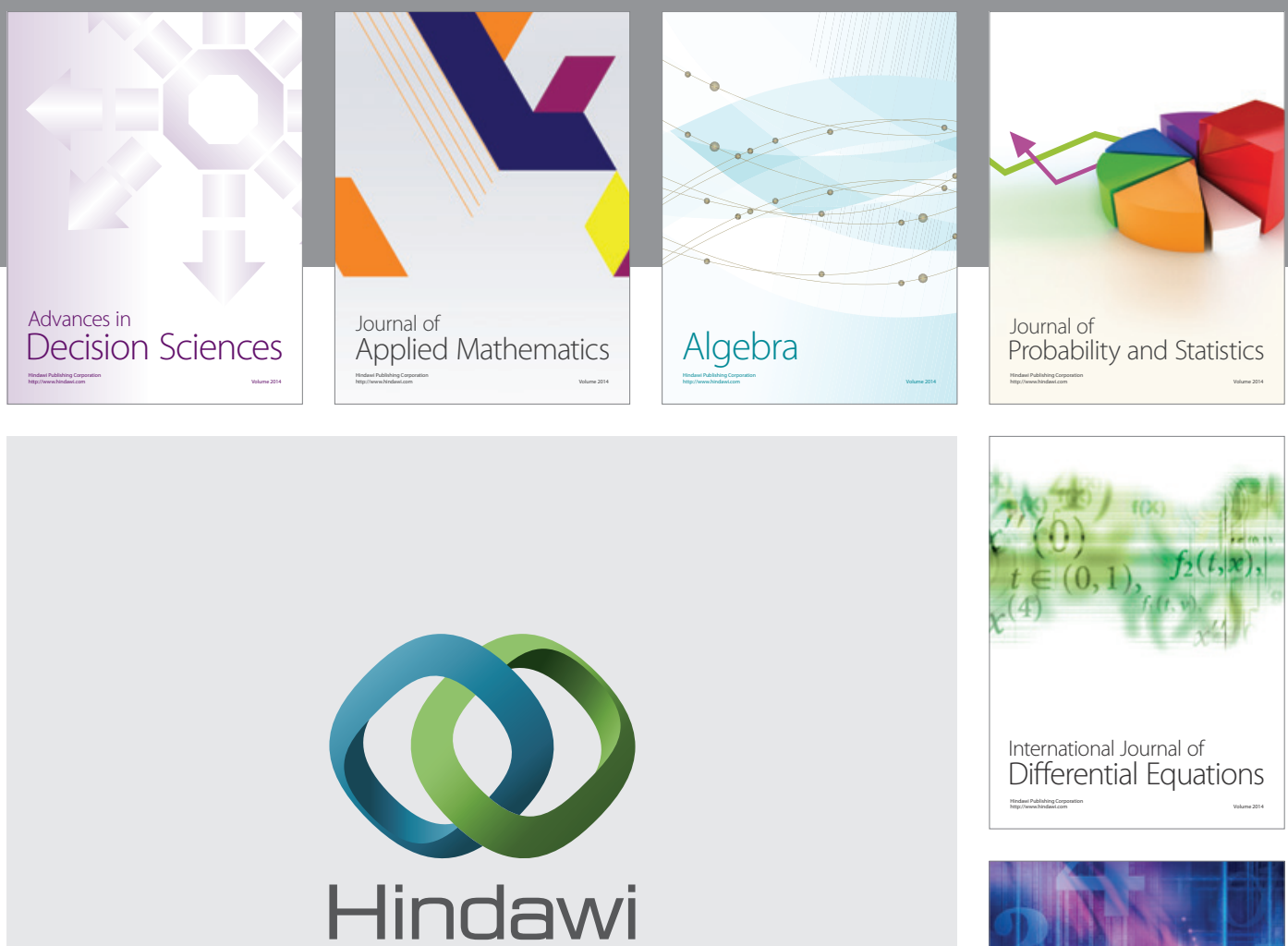

Submit your manuscripts at http://www.hindawi.com
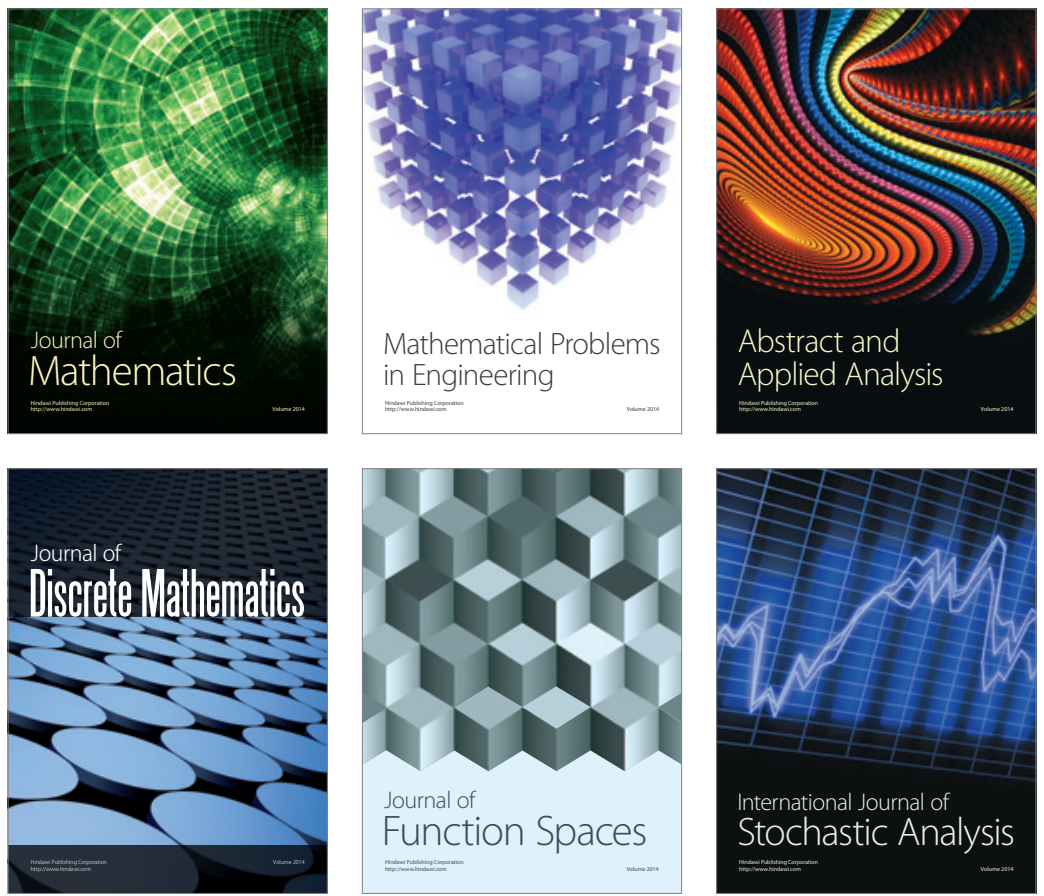

Journal of

Function Spaces

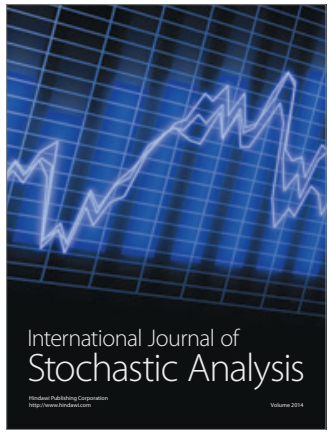

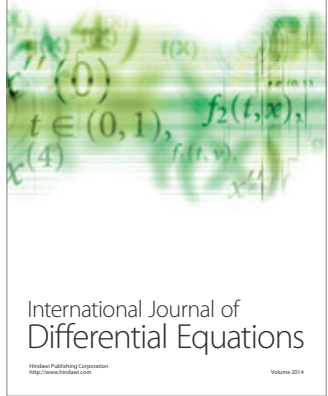
